\title{
Das Heilmittelgesetz und klinische Forschung
}

Das neue Heilmittelgesetz, das seit 1. Januar 2002 in Kraft ist, regelt neben Herstellung, Zulassung, Vertrieb und Abgabe von Heilmitteln auch die Durchführung von klinischen Versuchen. In diesem Bereich richtet sich das Gesetz im wesentlichen nach international anerkannten Standards wie die Good Clinical Practice. Neu geregelt sind auch die Verantwortlichkeiten und Kompetenzen zwischen den verschiedenen Akteuren der klinischen Forschung sowie zwischen Bund und Kantonen. Neben diesen eindeutigen Verbesserungen, die eine gesamtschweizerisch einheitliche Lösung in diesem bisher sehr zersplittert geregelten Bereich fast zwangsläufig mit sich bringen, bleiben aber weiterhin Probleme in Spannungsfeld zwischen Industrie und Forschung bestehen. Dominique Sprumont und Marie-Laure Béguin vom Institut de droit de la santé in Neuchâtel haben in einer Arbeit, die wir in dieser Ausgabe der SÄZ publizieren [1], diese Probleme im Detail untersucht. Probleme bestehen nach ihrer Ansicht vor allem noch im Bereich der Information und der Ausbildung der verschiedenen Akteure. Immerhin seien für die Ausbildung von Mitgliedern von Ethikkommissionen redliche Anstrengungen unternommen worden; erwähnt seien die Schweizerische Gesellschaft für biomedizinische Ethik, die SAMW, neuerdings auch die Kantone und Swissmedic, die entsprechende Ausbildungsangebote geschaffen haben. Hingegen sei die Ausbildung der Forscher, die in der Verantwortung der Industrie liege, noch als mangelhaft einzustufen. Wohl seien auch in diesem Bereich Ausbildungsangebote eingerichtet worden, z.B. von der Swiss Association of Pharmaceuticals Professionals (SWAPP) und vom European Center of Pharmaceutical Medicine (ECPM). Diese Angebote würden sich allerdings nur an ein kleines Publikum richten und könnten damit keinen ausreichenden Einfluss auf die Qualität von Forschungsprojekten erzielen. Gerade aber die Industrie, die sich gerne über administrative und gesetzliche Hürden beklagt, sollte nach Ansicht der Autoren ein Interesse an einer guten Ausbildung ihrer Forscher haben, weil sie damit viele ihrer Probleme von vornherein lösen könnte. Dies sei umso wichtiger, als die durch die Industrie finanzierte Forschung in den letzten Jahren sehr stark zugenommen habe. Deshalb sollten von den Ärzten, die an Forschungs- projekten teilnehmen, nicht nur medizinische, sondern gerade auch juristische Fachkenntnisse gefordert werden.

Allerdings wird es nicht damit getan sein, die Ausbildung zu fördern. Vielmehr werden weitere legislatorische Anstrengungen notwendig sein, um neue, durch die aktuelle Gesetzgebung nicht geregelte Probleme anzugehen. In diesem Zusammenhang ist darauf hinzuweisen, dass noch in diesem Jahr ein Gesetzesentwurf über die Forschung am Menschen in Vernehmlassung gehen soll. In diesem Gesetzeswerk wird unter anderem vorgeschlagen, den Forschern weitere Rechte zuzugestehen, wie die Mitbeteiligung bei der Gestaltung des Forschungsdesigns, der Zugang zur Gesamtheit der erhobenen Rohdaten und eine Garantie, die Resultate veröffentlichen zu dürfen. In einem viel beachteten Editorial $^{*}$ haben sich führende medizinische Fachzeitschriften dazu verpflichtet, nur noch Resultate zu veröffentlichen, die diesen Ansprüchen genügen.

Es ist zu hoffen, dass solche Forderungen rasch Eingang in die Gesetze finden werden. Es ist den Autoren beizupflichten, wenn sie als Schlussfolgerung ihrer Arbeit als Hauptziel die Wahrung der Integrität der Forschung nennen. Es geht darum, in allen Bereichen der Forschungsaktivitäten, insbesondere in finanziellen Belangen, transparente Verhältnisse zu schaffen. Nicht zuletzt muss auch die Rolle des Staates hinterfragt werden. Sprumont und Béguin mahnen, dass der Staat vordringlich die Unabhängigkeit und die hohe Qualität der Forschung zu garantieren habe, bevor er sich anschicke, Industrie und Forschung miteinander zu verbandeln. Letztlich geht es um den Schutz von Probanden und Patienten und auch darum, das Vertrauen der Öffentlichkeit in die Forschung zu erhalten. Vertrauen in diese Forschung aber ist unverzichtbare Voraussetzung für einen funktionierenden Forschungsplatz.

Dr. med. M. Trutmann

1 Sprumont D, Béguin M-L. La nouvelle réglementation des essais cliniques de médicaments. Schweiz Ärztezeitung 2002;83(18):894-906.

2 Smith R. Maintaining the integrity of the scientific record. Br Med J 2001;323:588. 